IMA Journal of Numerical Analysis (2014) 34, 675-699

doi:10.1093/imanum/drs059

Advance Access publication on July 14, 2013

\title{
Retarded boundary integral equations on the sphere: exact and numerical solution
}

\author{
S. SAUTER* AND A. VeIT \\ Institut für Mathematik, Universität Zürich, Winterthurerstrasse 190, CH-8057 Zürich, Switzerland \\ *Corresponding author: stas@math.uzh.ch alexander.veit@math.uzh.ch
}

[Received on 4 February 2012; revised on 26 October 2012]

\begin{abstract}
In this paper we consider the three-dimensional wave equation in unbounded domains with Dirichlet boundary conditions. We start from a retarded single-layer potential ansatz for the solution of these equations which leads to the retarded potential integral equation on the bounded surface of the scatterer. We formulate an algorithm for the space-time Galerkin discretization with smooth and compactly supported temporal basis functions, which were introduced in Sauter \& Veit (2013, Numer. Math., 145-176). For the debugging of an implementation and for systematic parameter tests it is essential to have at hand some explicit representations and some analytic properties of the exact solutions for some special cases. We will derive such explicit representations for the case where the scatterer is the unit ball. The obtained formulas are easy to implement and we will present some numerical experiments for these cases to illustrate the convergence behaviour of the proposed method.
\end{abstract}

Keywords: retarded potentials; space-time Galerkin method; exact solution; boundary integral equations; three-dimensional wave equation.

\section{Introduction}

Mathematical modelling of acoustic and electromagnetic wave propagation and its efficient and accurate numerical simulation is a key technology for numerous engineering applications as, for example, in detection (nondestructive testing, radar), communication (optoelectronic and wireless) and medicine (sonic imaging, tomography). An adequate model problem for the development of efficient numerical methods for such types of physical applications is the three-dimensional wave equation in unbounded exterior domains. In this setting the method of integral equations is an elegant approach since it reduces the problem in the unbounded domain to a retarded potential integral equation (RPIE) on the bounded surface of the scatterer.

In the literature there exist different approaches for the numerical discretization of these retarded boundary integral equations. They include collocation schemes with some stabilization techniques (cf. Rynne \& Smith, 1990; Bluck \& Walker, 1996; Davies \& Duncan, 1997, 2003; Dodson et al., 1997; Birgisson et al., 1999), methods based on bandlimited interpolation and extrapolation (cf. Weile et al., 2000, 2001, 2004; Wildman et al., 2004), convolution quadrature (cf. Hackbusch et al., 2007, 2009; Banjai \& Sauter, 2008; Wang et al., 2008; Banjai, 2010; Banjai et al., 2011; López-Fernández \& Sauter, 2013; Banjai \& Schanz, 2012), as well as methods using space-time integral equations (cf. Bamberger \& Duong, 1986; Ding et al., 1989; Ha-Duong, 2003; Ha-Duong et al., 2003; Sauter \& Veit, 2013).

In Sauter \& Veit (2013) and Veit (2012) a space-time Galerkin method for the discretization of RPIEs with smooth and compactly supported temporal basis functions was introduced conceptually. In this paper we present an algorithmic formulation of the method. The debugging of the implementation 
and the investigation of the performance and sensitivity of the method with respect to various parameters require a careful implementation and some exact solutions for performing appropriate numerical experiments. It turns out that the derivation of an explicit representation of the solution for some special geometry (here, the unit sphere in $\mathbb{R}^{3}$ ) and Dirichlet boundary conditions is by no means trivial. We start from a retarded single-layer potential ansatz for the solution of this equation which results in a retarded boundary integral equation on the sphere with unknown density function $\phi$. We use Laplace transformations in order to transfer these problems to univariate problems in time which we solve analytically. The obtained explicit formulas for $\phi$ lead to exact solutions of the full scattering problem on the sphere and they are easy to implement. We employ these reference solutions for verifying the accuracy of our new method by numerical experiments and for studying its performance and convergence behaviour. Furthermore, these formulas are suitable for studying analytic properties of these density functions.

An easy-to-use MATLAB script is available at https://www.math.uzh.ch/compmath/?exactsolutions, which implements the formulas obtained in this article.

\section{Integral formulation of the wave equation}

Let $\Omega \subset \mathbb{R}^{3}$ be a Lipschitz domain with boundary $\Gamma$. We consider the homogeneous wave equation

$$
\partial_{t}^{2} u-\Delta u=0 \quad \text { in } \Omega \times[0, T]
$$

with initial conditions

$$
u(\cdot, 0)=\partial_{t} u(\cdot, 0)=0 \quad \text { in } \Omega
$$

and Dirichlet boundary conditions

$$
u=g \quad \text { on } \Gamma \times[0, T]
$$

on a time interval $[0, T]$ for $T>0$. In applications, $\Omega$ is often the unbounded exterior of a bounded domain. For such problems, the method of boundary integral equations is an elegant tool where this partial differential equation is transformed into an equation on the bounded surface $\Gamma$. We employ an ansatz as a single-layer potential for the solution $u$,

$$
u(x, t):=S \phi(x, t):=\int_{\Gamma} \frac{\phi(y, t-\|x-y\|)}{4 \pi\|x-y\|} \mathrm{d} \Gamma_{y}, \quad(x, t) \in \Omega \times[0, T],
$$

with unknown density function $\phi$. We also refer to $S$ as a retarded single-layer potential due to the retarded time argument $t-\|x-y\|$ which connects time and space variables.

The ansatz (2.2) satisfies the wave equation (2.1a) and the initial conditions (2.1b). Since the singlelayer potential can be extended continuously to the boundary $\Gamma$, the unknown density function $\phi$ is determined such that the boundary conditions (2.1c) are satisfied. This results in the boundary integral equation for $\phi$,

$$
\int_{\Gamma} \frac{\phi(y, t-\|x-y\|)}{4 \pi\|x-y\|} \mathrm{d} \Gamma_{y}=g(x, t) \quad \forall(x, t) \in \Gamma \times[0, T] .
$$

Existence and uniqueness results for the solution of the continuous problem are proved in Lubich (1994). 


\section{Temporal Galerkin discretization of retarded potentials with smooth basis functions}

In this section we recall the Galerkin discretization of the boundary integral equation (2.3) using smooth and compactly supported basis functions in time. For details and an analysis of the scheme we refer the reader to Sauter \& Veit (2013).

A coercive space-time variational formulation of (2.3) is given by the following (cf. Bamberger \& Duong, 1986; Ha-Duong, 2003): find $\phi$ in an appropriate Sobolev space $V$ such that

$$
\int_{0}^{T} \int_{\Gamma} \int_{\Gamma} \frac{\dot{\phi}(y, t-\|x-y\|) \zeta(x, t)}{4 \pi\|x-y\|} \mathrm{d} \Gamma_{y} \mathrm{~d} \Gamma_{x} \mathrm{~d} t=\int_{0}^{T} \int_{\Gamma} \dot{g}(x, t) \zeta(x, t) \mathrm{d} \Gamma_{x} \mathrm{~d} t
$$

for all $\zeta \in V$, where we denote by $\dot{\phi}$ the derivative with respect to time. The Galerkin discretization of (3.1) now consists of replacing $V$ by a finite-dimensional subspace $V_{\text {Galerkin }}$ being spanned by $L$ basis functions $\left\{b_{i}\right\}_{i=1}^{L}$ in time and $M$ basis functions $\left\{\varphi_{j}\right\}_{j=1}^{M}$ in space. This leads to the discrete ansatz

$$
\phi_{\text {Galerkin }}(x, t)=\sum_{i=1}^{L} \sum_{j=1}^{M} \alpha_{i}^{j} \varphi_{j}(x) b_{i}(t), \quad(x, t) \in \Gamma \times[0, T],
$$

for the approximate solution, where $\alpha_{i}^{j}$ are the unknown coefficients. As mentioned above we will use smooth and compactly supported temporal shape functions $b_{i}$ in (3.2). Their definition was addressed in Sauter \& Veit (2013) and is as follows. Let

$$
f(t):= \begin{cases}\frac{1}{2} \operatorname{erf}(2 \operatorname{arctanh} t)+\frac{1}{2}, & |t|<1 \\ 0, & t \leqslant-1 \\ 1, & t \geqslant 1\end{cases}
$$

and note that $f \in C^{\infty}(\mathbb{R}) .{ }^{1}$ Next, we will introduce some scaling. For a function $g \in C^{0}([-1,1])$ and real numbers $a<b$, we define $g_{a, b} \in C^{0}([a, b])$ by

$$
g_{a, b}(t):=g\left(2 \frac{t-a}{b-a}-1\right) .
$$

We obtain a bump function on the interval $[a, c]$ with joint $b \in(a, c)$ :

$$
\rho_{a, b, c}(t):= \begin{cases}f_{a, b}(t), & a \leqslant t \leqslant b, \\ 1-f_{b, c}(t), & b \leqslant t \leqslant c, \\ 0, & \text { otherwise. }\end{cases}
$$

Let us now consider the closed interval $[0, T]$ and $l$ (not necessarily equidistant) time steps

$$
0=t_{0}<t_{1}<\cdots<t_{l-2}<t_{l-1}=T \text {. }
$$

\footnotetext{
1 Note that this choice of $f$ is by no means unique. In Chernov et al. $(2011$, Section 6.1$), C^{\infty}(\mathbb{R})$ bump functions are considered (in a different context) which have certain Gevrey regularity. They also could be used for our partition of unity.
} 
A smooth partition of unity of the interval $[0, T]$ then is defined by

$$
\mu_{1}:=1-f_{t_{0}, t_{1}} ; \quad \mu_{l}:=f_{t_{l-2, l-1}} ; \quad \forall 2 \leqslant i \leqslant l-1: \mu_{i}:=\rho_{t_{i-2}, t_{i-1}, t_{i}} .
$$

Smooth and compactly supported basis functions $b_{i}$ in time can then be obtained by multiplying these partition-of-unity functions with suitably scaled Legendre polynomials (see Sauter \& Veit, 2013 for details).

For the discretization in space we use standard piecewise polynomial basis functions $\varphi_{j}$. The solution of (3.1) using the discrete ansatz (3.2) leads to a linear system with $L \cdot M$ unknowns. We partition the resulting system matrix $\underline{\underline{\mathbf{A}}}$ and right-hand side $\underline{\mathbf{g}}$ as a block matrix/block vector according to

$$
\underline{\underline{\mathbf{A}}}:=\left[\begin{array}{cccc}
\mathbf{A}_{1,1} & \mathbf{A}_{1,2} & \cdots & \mathbf{A}_{1, L} \\
\mathbf{A}_{2,1} & \mathbf{A}_{2,2} & \cdots & \mathbf{A}_{2, L} \\
\vdots & \vdots & \ddots & \vdots \\
\mathbf{A}_{L, 1} & \mathbf{A}_{L, 2} & \cdots & \mathbf{A}_{L, L}
\end{array}\right], \quad \underline{\mathbf{g}}:=\left[\begin{array}{c}
\mathbf{g}_{1} \\
\mathbf{g}_{2} \\
\vdots \\
\mathbf{g}_{L}
\end{array}\right],
$$

where

$$
\mathbf{A}_{k, i} \in \mathbb{R}^{M \times M}, \quad \mathbf{g}_{k} \in \mathbb{R}^{M} \quad \text { for } i, k \in\{1, \ldots, L\} .
$$

Furthermore, we denote

$$
\min _{k}:=\min \operatorname{supp} b_{k}, \quad \max _{k}:=\max \operatorname{supp} b_{k},
$$

for $k \in\{1, \ldots, L\}$. Algorithm 1 computes the unknown coefficients $\alpha_{i}^{j}$ in (3.2) and leads to a solution of the boundary integral equation (2.3).

Algorithm 1 Computation of the coefficients $\alpha_{i}^{j}$ in (3.2)

Input: - A triangulation $\mathcal{G}:=\left\{\tau_{i}: 1 \leqslant i \leqslant \bar{M}\right\}$ of $\Gamma$ consisting of (possibly curved) triangles $\tau_{i}$.

- $L$ : number of basis functions in time (defined on a not necessarily equidistant time grid).

- Time derivative $\dot{g}(x, t)$ of right-hand side.

$\{$ Generation of right-hand side $\}$

for $k=1$ to $L$ do

$$
\mathbf{g}_{k} \leftarrow\left(\int_{0}^{T} \int_{\Gamma} \dot{g}(x, t) \varphi_{l}(x) b_{k}(t) \mathrm{d} \Gamma_{x} \mathrm{~d} t\right)_{l=1}^{M} \in \mathbb{R}^{M}
$$

\{Blockwise generation of system matrix $\}$

for $i=1$ to $L$ do

if $\min _{i} \geqslant \max _{k}$ then

$$
\mathbf{A}_{k, i} \leftarrow \mathbf{0} \in \mathbb{R}^{M \times M}
$$

else

for $j, l=1$ to $M$ do

mindist $\leftarrow \operatorname{dist}\left(\operatorname{supp} \varphi_{j}, \operatorname{supp} \varphi_{l}\right)$

$\operatorname{maxdist} \leftarrow \sup _{(x, y) \in \operatorname{Supp} \varphi_{l} \times \operatorname{Supp} \varphi_{j}}\|x-y\|$ 


$$
\text { if }\left[\min _{k}-\max _{i}, \max _{k}-\min _{i}\right] \cap[\text { mindist, maxdist }]=\emptyset \text { then }
$$

$$
\mathbf{A}_{k, i}(j, l) \leftarrow 0 \in \mathbb{R}
$$

else

$$
\mathbf{A}_{k, i}(j, l) \leftarrow \int_{0}^{T} \int_{\Gamma} \int_{\Gamma} \frac{\varphi_{j}(y) \varphi_{l}(x)}{4 \pi\|x-y\|} \dot{b}_{i}(t-\|x-y\|) b_{k}(t) \mathrm{d} \Gamma_{y} \mathrm{~d} \Gamma_{x} \mathrm{~d} t
$$

\section{end if \\ end for \\ end if \\ end for \\ end for}

\{Solution of linear system $\}$

Solve:

$$
\underline{\underline{\mathbf{A}}} \cdot \underline{\mathbf{x}}=\underline{\mathbf{g}} \quad \text { with } \mathbf{x} \in \mathbb{R}^{L M}
$$

Output: The vector $\underline{\mathbf{x}}$ corresponds to the unknown coefficients in (3.2).

REMARK 3.1 (Numerical quadrature) The most time-consuming part of this algorithm is the computation of the matrix entries $\mathbf{A}_{k, i}(j, l)$ by numerical quadrature. Define

$$
\psi_{k, i}(r)=\int_{0}^{T} \dot{b}_{i}(t-r) b_{k}(t) \mathrm{d} t=\int_{\min _{k}}^{\max _{k}} \dot{b}_{i}(t-r) b_{k}(t) \mathrm{d} t,
$$

with supp $\psi_{k, i}=\left[\min _{k}-\max _{i}, \max _{k}-\min _{i}\right]$. Then, we can rewrite (3.6) as

$$
\mathbf{A}_{k, i}(j, l)=\int_{\Gamma} \int_{\Gamma} \frac{\varphi_{j}(y) \varphi_{l}(x)}{4 \pi\|x-y\|} \psi_{k, i}(\|x-y\|) \mathrm{d} \Gamma_{y} \mathrm{~d} \Gamma_{x} .
$$

In order to compute integrals of the form (3.8) the regularizing coordinate transform as explained in Sauter \& Schwab (2011) can be applied. This transform removes the spatial singularity at $x=y$ via the determinant of the Jacobian. The resulting integration domain is the four-dimensional unit cube. In order to approximate the transformed integrals, tensor-Gauss quadrature can be used. Note that the integrands in (3.8) are $C^{\infty}$ smooth but not analytic and therefore classical error estimates are not valid. In Sauter \& Veit (2013) we have developed a quadrature error analysis for this type of integrand. Instead of tensor-Gauss quadrature it might also be suitable to use other quadrature schemes like sparse grid or adaptive quadrature in order to reduce the computational complexity. In Khoromskij et al. (2011) we proposed a method based on sparse tensor approximation to evaluate these integrals.

For the approximation of the matrix entries (3.8) the function $\psi_{k, i}$ has to be evaluated multiple times. Since such an evaluation by a quadrature rule is costly we suggest approximating $\psi_{k, i}$ accurately on its support by a polynomial (e.g., by interpolation), which can be evaluated efficiently. Here, we use accurate piecewise interpolation at Chebyshev nodes. Compared with a five-dimensional numerical integration in (3.8) this approach leads in practice to more accurate results at lower computational cost. 

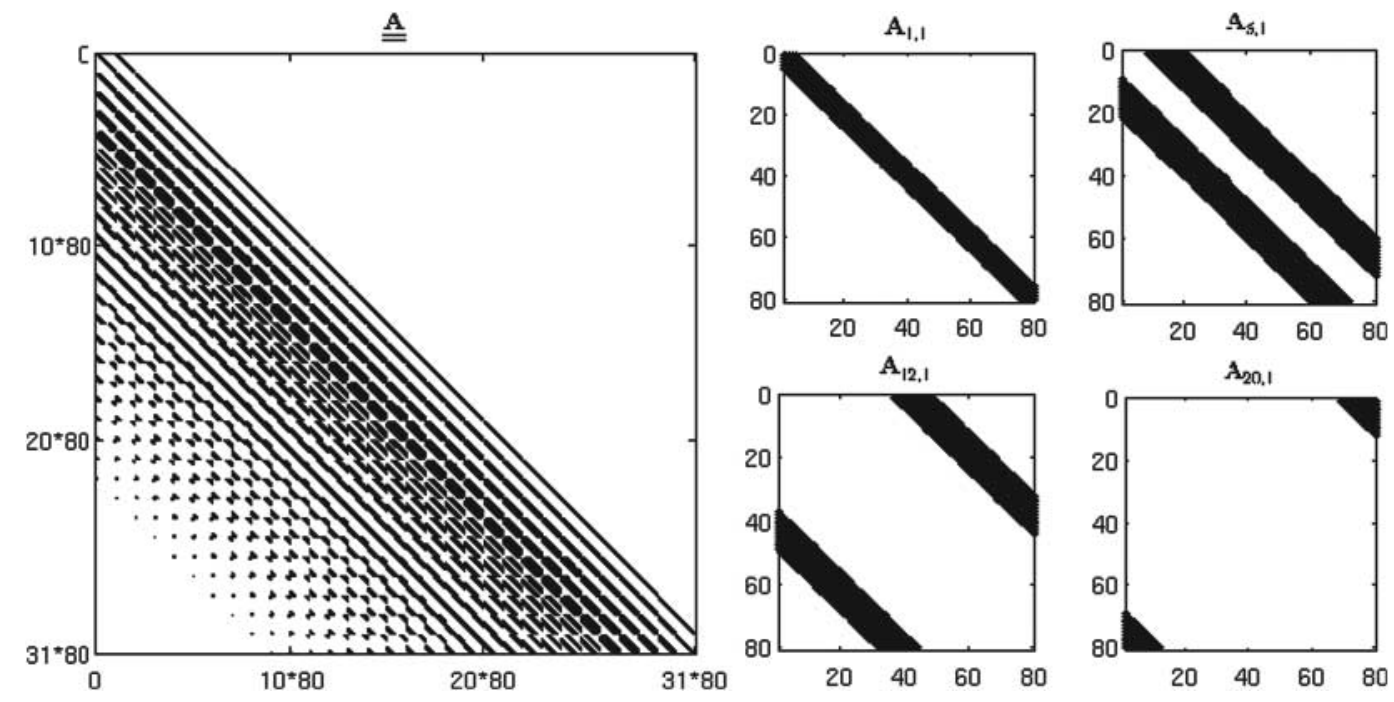

FIG. 1. Sparsity pattern of the matrix $\underline{\underline{\mathbf{A}}}$ and its blocks $\mathbf{A}_{k, i}$.

REMARK 3.2 (Sparsity pattern of the matrix) The matrix $\underline{\underline{\mathbf{A}}}$ in (3.3) is a block matrix where the lower triangular part in general is nonzero while, according to (3.4) and (3.5), only very few upper off-diagonals are nonvanishing. The matrix blocks $\mathbf{A}_{k, i}$ in general are sparse-only the entries which are enlighted by the support of the relevant temporal basis functions are nonzero.

In order to estimate the number of nonzero entries in $\underline{\mathbf{A}}$ we consider for simplicity a quasi-uniform spatial mesh with mesh width $h$ and $M \sim h^{-2}$ degrees of freedom and $L$ basis functions in time.

(a) Equidistant time grid with step size $\Delta t$. Since in this case $\left|\operatorname{supp} \psi_{k, i}\right|=\mathcal{O}(\Delta t)$, the number of nonzero entries in the matrix block $\mathbf{A}_{k, i}$ can be estimated by $M\left(\left(\Delta t+h^{2}\right) / h^{2}\right)$. Since in the case of equidistant time steps we have only $\mathcal{O}(L)$ different matrix blocks, this sums to $\mathcal{O}\left(M^{2}+L M\right)$ entries of $\underline{\underline{\mathbf{A}}}$ that have to be computed.

(b) Quasi-uniform, nonequidistant time steps $\left\{t_{i}\right\}_{i=0}^{l-1}$ with step size $\Delta_{i}:=t_{i}-t_{i-1}$. The number of nonzero entries in the matrix block $\mathbf{A}_{k, i}$ can be estimated by $M\left(\left(\Delta_{i}+\Delta_{k}+h^{2}\right) / h^{2}\right)$. In this case the computational and storage costs sum to $\mathcal{O}\left(M^{2} L+M L^{2}\right)$.

In Fig. 1 the sparsity pattern of $\underline{\underline{\mathbf{A}}}$ and its matrix blocks are depicted. For illustrative purposes, we choose $\Gamma$ to be the one-dimensional interval $[0,2]$, subdivided into 80 equidistant subintervals, and the time interval to be $[0,3]$, subdivided into 30 equidistant subintervals. As temporal basis functions we use the smooth partition of unity described above.

\section{Exact solutions of the wave equation for $\Gamma=\mathbb{S}^{2}$}

The systematic numerical testing of the convergence behaviour of our discretization requires knowledge of exact solutions for some specific model problems whose derivation is far from trivial. Hence, a substantial part of this paper is devoted to the derivation of such solutions for a spherical scatterer. In Section 5 we shall report on the approximation of these solutions by our method. 
In this section we will derive analytic solutions of (2.3) for the special case where the boundary of the scatterer $\Gamma$ is the unit sphere in $\mathbb{R}^{3}$. Note that an equivalent formulation of the retarded single-layer potential (2.2) is given by

$$
S \phi(x, t)=\int_{0}^{t} \int_{\Gamma} k(x-y, t-\tau) \phi(y, \tau) \mathrm{d} \Gamma_{y} \mathrm{~d} \tau, \quad(x, t) \in \Omega \times[0, T],
$$

where $k(z, t)$ is the fundamental solution of the wave equation,

$$
k(z, t)=\frac{\delta(t-\|z\|)}{4 \pi\|z\|}
$$

$\delta(t)$ being the Dirac delta distribution. This representation is usually the starting point of discretization methods based on convolution quadrature, where only the Laplace transform of the kernel function is used. We introduce the single-layer potential for the Helmholtz operator $\Delta U-s^{2} U=0$ which is given by

$$
(V(s) \varphi)(x):=\int_{\Gamma} K(s, x-y) \varphi(y, \tau) \mathrm{d} \Gamma_{y}, \quad x \in \mathbb{R}^{3}
$$

where

$$
K(s, z):=\frac{e^{-s\|z\|}}{4 \pi\|z\|}
$$

is the fundamental solution of the Helmholtz equation in three spatial dimensions. We now adopt the setting in Banjai \& Sauter (2008). We want to solve the boundary integral equation (2.3) in the case where $\Gamma$ is the unit sphere $\mathbb{S}^{2}$. For the right-hand side $g$ we assume causality; that is, $g(x, t)=0$ for $t \leqslant 0$ and, furthermore, that at least the first time derivative of $g$ vanishes at $t=0$. Moreover, $g$ is supposed to be of the form

$$
g(x, t)=g(t) Y_{n}^{m},
$$

where $Y_{n}^{m}$ denotes a spherical harmonic of degree $n$ and order $m$. The $Y_{n}^{m}$ are eigenfunctions of the single-layer potential for the Helmholtz operator, that is,

$$
V(s) Y_{n}^{m}=\lambda_{n}(s) Y_{n}^{m},
$$

with eigenvalues $\lambda_{n}(s)$.

REMARK 4.1 The availability of eigenfunctions and eigenvalues of the frequency domain operator is crucial for the computation of exact solutions of (2.3). We refer the reader to Kress (1985) and Nédélec (2001) for the derivation of those in the case of the single-layer potential for the stationary Helmholtz equation. For the double-layer potential, the adjoint double-layer potential and the hypersingular operator in the frequency domain, similar formulas exist (cf. Nédélec, 2001). In the same way as described below we can therefore obtain exact solutions also for other time-domain boundary integral equations arising in Dirichlet and Neumann problems in acoustic scattering. Details and explicit formulas for other problems can be found in Veit (2012). 
We express the eigenvalues $\lambda_{n}(s)$ in terms of modified Bessel functions $I_{\kappa}$ and $K_{\kappa}$ (see Abramowitz \& Stegun, 1972):

$$
\lambda_{n}(s)=I_{n+1 / 2}(s) K_{n+1 / 2}(s) .
$$

Next, we will reduce equation (2.3) to a univariate problem in time. Recall the definition of the Laplace transform,

$$
\hat{\phi}(s):=(\mathcal{L} \phi)(s)=\int_{0}^{\infty} \phi(t) e^{-s t} \mathrm{~d} t,
$$

with inverse

$$
\left(\mathcal{L}^{-1} \hat{\phi}\right)(s)=\frac{1}{2 \pi \mathrm{i}} \int_{\sigma-\mathrm{i} \infty}^{\sigma+\mathrm{i} \infty} \hat{\phi}(s) e^{s t} \mathrm{~d} s \quad \text { for some } \sigma>0
$$

Note that the fundamental solution of the Helmholtz equation is the Laplace transform of the fundamental solution of the wave equation. Using the representation (4.1) for $S$ and expressing $k$ in terms of its Laplace transform leads to the integral equation

$$
\begin{aligned}
g(t) Y_{n}^{m} & =\int_{0}^{t} \int_{\Gamma} k(t-\tau,\|x-y\|) \phi(y, \tau) \mathrm{d} \Gamma_{y} \mathrm{~d} \tau \\
& =\frac{1}{2 \pi \mathrm{i}} \int_{\sigma-\mathrm{i} \infty}^{\sigma+\mathrm{i} \infty} \int_{0}^{t} e^{s \tau} \int_{\Gamma} K(s,\|x-y\|) \phi(y, t-\tau) \mathrm{d} \Gamma_{y} \mathrm{~d} \tau \mathrm{d} s \\
& =\frac{1}{2 \pi \mathrm{i}} \int_{\sigma-\mathrm{i} \infty}^{\sigma+\mathrm{i} \infty} \int_{0}^{t} e^{s \tau}(V(s) \phi(\cdot, t-\tau))(x) \mathrm{d} \tau \mathrm{d} s .
\end{aligned}
$$

Inserting the ansatz $\phi(x, t)=\phi(t) Y_{n}^{m}$ into (4.2) leads to the following one-dimensional problem: find $\phi(t)$ such that

$$
g(t)=\int_{0}^{t} \mathcal{L}^{-1}\left(\lambda_{n}\right)(\tau) \phi(t-\tau) \mathrm{d} \tau
$$

Applying the Laplace transformation to both sides yields

$$
\hat{g}(s)=\lambda_{n}(s) \hat{\phi}(s) .
$$

Rearranging terms and applying an inverse Laplace transformation finally leads to an expression for $\phi$ :

$$
\phi(t)=\int_{0}^{t} g(\tau) \mathcal{L}^{-1}\left(\frac{1}{\lambda_{n}}\right)(t-\tau) \mathrm{d} \tau .
$$

Note that $\phi(t) Y_{n}^{m}$ with $\phi(t)$ as above is a solution of the full problem (2.3) in the case where $\Gamma=\mathbb{S}^{2}$ and $g(x, t)=g(t) Y_{n}^{m}$.

Before we proceed with the computation of (4.4), note that, with the above formulas, it is also possible to find an expression for the solution $\phi(x, t)$ in $(2.3)$ for more general right-hand sides. If we choose the normalization convention for $Y_{n}^{m}$ such that they form an orthonormal system in $L^{2}\left(\mathbb{S}^{2}\right)$ : $\left(Y_{n}^{m}, Y_{n^{\prime}}^{m^{\prime}}\right)_{L^{2}\left(\mathbb{S}^{2}\right)}=\delta_{n, n^{\prime}} \delta_{m, m^{\prime}}$, the following theorem holds. 
TheOrem 4.2 Let the right-hand side of (2.3) be causal, that is, $g(x, t)=0$ for $t \leqslant 0$, for all $x \in \mathbb{S}^{2}$ and assume that $\partial_{t} g(x, 0)=0$, for all $x \in \mathbb{S}^{2}$. Let $g$ be of the form

$$
g(x, t)=\sum_{n=0}^{\infty} \sum_{m=-n}^{n} g_{n, m}(t) Y_{n}^{m}
$$

Then, the solution $\phi$ has the form

$$
\phi(x, t)=\sum_{n=0}^{\infty} \sum_{m=-n}^{n} \phi_{n, m}(t) Y_{n}^{m}
$$

where

$$
\phi_{n, m}=\int_{0}^{t} g_{n, m}(\tau) \mathcal{L}^{-1}\left(\frac{1}{\lambda_{n}}\right)(t-\tau) \mathrm{d} \tau .
$$

Note that the expressions in Theorem 4.2 are considered as formal series. However, the existence and uniqueness results in Ha-Duong (2003) imply that, for a given right-hand side $g$ with $\dot{g} \in$ $H^{1 / 2,1 / 2}(\Gamma \times[0, T]):=L^{2}\left(0, T ; H^{1 / 2}(\Gamma)\right) \cap H^{1 / 2}\left(0, T ; L^{2}(\Gamma)\right)$, the solution $\phi$ exists in $H^{-1 / 2,-1 / 2}(\Gamma \times$ $[0, T]):=L^{2}\left(0, T ; H^{-1 / 2}(\Gamma)\right)+H^{-1 / 2}\left(0, T ; L^{2}(\Gamma)\right)$.

If only finitely many Fourier coefficients of $g$ are nonzero, then the expansion of $\phi$ and the existence in the classical pointwise sense is obvious.

For simplicity we return to the situation in (4.4) where we consider only one mode of such an expansion. In order to find an analytic expression for $\phi(t)$, it is necessary to find a representation for the inverse Laplace transform of $1 / \lambda_{n}(s)$. With the formulas in Gradshteyn \& Ryzhik (1965, Sections 8.467 and 8.468) we get

$$
\lambda_{n}(s)=I_{n+1 / 2}(s) K_{n+1 / 2}(s)=\frac{y_{n}(-1 / s) y_{n}(1 / s)+(-1)^{n+1} y_{n}^{2}(1 / s) e^{-2 s}}{2 s},
$$

where

$$
y_{n}(s):=\sum_{k=0}^{n}(n, k) s^{k} \quad \text { with } \quad(n, k):=\frac{(n+k) !}{2^{k} k !(n-k) !}
$$

are the Bessel polynomials (see Ismail, 2009, Section 4.10). This is equivalent to

$$
\lambda_{n}(s)=(-1)^{n} \frac{\theta_{n}(s)}{2 s^{2 n+1}}\left(\theta_{n}(-s)-\theta_{n}(s) e^{-2 s}\right),
$$

where $\theta_{n}$ are the reversed Bessel polynomials

$$
\theta_{n}(s):=\sum_{k=0}^{n}(n, k) s^{n-k}
$$

After some manipulation we therefore get for the inverse Laplace transform,

$$
\mathcal{L}^{-1}\left(\frac{1}{\lambda_{n}}\right)=2 \delta^{\prime}+(-1)^{n} 2 \partial_{t} \mathcal{L}^{-1}\left(\frac{\tilde{\theta}_{2 n-2}(s)+(-1)^{n} \theta_{n}(s)^{2} e^{-2 s}}{\theta_{n}(-s) \theta_{n}(s)-\theta_{n}(s)^{2} e^{-2 s}}\right),
$$


where

$$
\mathbb{P}_{\max (0,2 n-2)} \ni \tilde{\theta}_{2 n-2}(s)=s^{2 n}-(-1)^{n} \theta_{n}(-s) \theta_{n}(s) .
$$

We expand the term in the brackets in the right-hand side of (4.6) with respect to $\varepsilon=e^{-2 s}$ about 0 and obtain

$$
\frac{\tilde{\theta}_{2 n-2}(s)+(-1)^{n} \theta_{n}(s)^{2} e^{-2 s}}{\theta_{n}(-s) \theta_{n}(s)-\theta_{n}(s)^{2} e^{-2 s}}=\underbrace{\frac{\tilde{\theta}_{2 n-2}(s)}{\theta_{n}(-s) \theta_{n}(s)}}_{R_{n}^{(1)}}+\sum_{k=1}^{\infty}\{(-1)^{n} \frac{\theta_{n}(s)^{k}}{\underbrace{(-s)^{k}}_{R_{n, k}^{(2)}} e^{-2 k s}}+\underbrace{\frac{\tilde{\theta}_{2 n-2}(s) \theta_{n}(s)^{k-1}}{\theta_{n}(-s)^{k+1}} e^{-2 k s}}_{R_{n, k}^{(3)}}\} .
$$

The computation of the inverse Laplace transforms of $R_{n}^{(1)}, R_{n, k}^{(2)}$ and $R_{n, k}^{(3)}$ boils down to the inversion of rational functions. This is done with the formulas in Erdélyi et al. (1954, Section 5.2). Note that $\theta_{n}(s)$ is a polynomial of degree $n$ and has exactly $n$ complex-valued simple zeros (cf. Ismail, 2009). Let

$$
\theta_{n}\left(\alpha_{i}\right)=0 \quad \text { for } i=1, \ldots, n \quad \text { where } \alpha_{i}=\alpha_{i}^{\mathrm{re}}+\mathrm{i} \alpha_{i}^{\mathrm{im}} \quad \text { with } \alpha_{i}^{\mathrm{re}}, \alpha_{i}^{\mathrm{im}} \in \mathbb{R} .
$$

It follows that the zeros of $\theta_{n}(-s)$ are $-\alpha_{1}, \ldots,-\alpha_{n}$. Thus, we get

$$
\mathcal{L}^{-1}\left(R_{n}^{(1)}\right)(t)=\sum_{j=1}^{n} c_{n, j}^{(1)} e^{\alpha_{j} t}+\tilde{c}_{n, j}^{(1)} e^{-\alpha_{j} t},
$$

where $c_{n, j}^{(1)}$ and $\tilde{c}_{n, j}^{(1)}$ are the coefficients of the partial fraction decomposition of $R_{n}^{(1)}$. Since the solution $\phi$ is real, we may restrict our consideration to the real part of $\mathcal{L}^{-1}\left(R_{n}^{(1)}\right)$. We denote the real part of $c_{n, j}^{(1)}$ by $c_{n, j}^{(1), \text { re }}$ and its imaginary part by $c_{n, j}^{(1), \text { im }}$. The notation for $\tilde{c}_{n, j}^{(1)}$ is chosen accordingly. We get

$$
\begin{aligned}
\mathcal{L}_{\mathrm{re}}^{-1}\left(R_{n}^{(1)}\right)(t)= & \sum_{j=1}^{n} c_{n, j}^{(1), \mathrm{re}} e^{\alpha_{j}^{\mathrm{re}} t} \cos \left(\alpha_{j}^{\mathrm{im}} t\right)-c_{n, j}^{(1), \mathrm{im}} e^{\alpha_{j}^{\mathrm{re}} t} \sin \left(\alpha_{j}^{\mathrm{im}} t\right) \\
& +\tilde{c}_{n, j}^{(1), \mathrm{re}} e^{-\alpha_{j}^{\mathrm{re}} t} \cos \left(-\alpha_{j}^{\mathrm{im}} t\right)-\tilde{c}_{n, j}^{(1), \mathrm{im}} e^{-\alpha_{j}^{\mathrm{re}} t} \sin \left(-\alpha_{j}^{\mathrm{im}} t\right) .
\end{aligned}
$$

REMARK 4.3 The coefficients $c_{n, j}^{(1)}$ and $\tilde{c}_{n, j}^{(1)}$ come in complex conjugate pairs. This could be exploited in the formula above. However, in order to keep the presentation as simple as possible we will not make use of this fact here.

REMARK 4.4 In Sections 4.1 and 4.2 we will state explicit representations of $\phi$ for $n=0,1$. In this case the above formula simplifies considerably. We get

$$
\mathcal{L}_{\mathrm{re}}^{-1}\left(R_{0}^{(1)}\right)(t)=0
$$

and

$$
\mathcal{L}_{\mathrm{re}}^{-1}\left(R_{1}^{(1)}\right)(t)=\frac{1}{2}\left(e^{-t}-e^{t}\right)=-\sinh (t) .
$$

For larger $n$ the coefficients arising from the inversions can be easily computed with computer algebra systems (see https://www.math.uzh.ch/compmath/?exactsolutions for a MATLAB implementation). 
For the computation of $\mathcal{L}^{-1}\left(R_{n, k}^{(2)}\right)$ we use the time-shifting property of the Laplace transformation. We employ the Heaviside step function

$$
H(t)= \begin{cases}0, & t \leqslant 0 \\ 1, & t>0\end{cases}
$$

to obtain

$$
\begin{aligned}
\mathcal{L}^{-1}\left(R_{n, k}^{(2)}\right)(t) & =\mathcal{L}^{-1}\left(\frac{\theta_{n}(s)^{k}}{\theta_{n}(-s)^{k}} e^{-2 k s}\right)(t)=H(t-2 k) \mathcal{L}^{-1}\left(\frac{\theta_{n}(s)^{k}}{\theta_{n}(-s)^{k}}\right)(t-2 k) \\
& =(-1)^{n k} \delta(t-2 k) H(t-2 k)+\sum_{i=1}^{n} \sum_{j=1}^{k} c_{n, k, j, i}^{(2)} H(t-2 k)(t-2 k)^{j-1} e^{-\alpha_{i}(t-2 k)},
\end{aligned}
$$

with some complex coefficients $c_{n, k, j, i}^{(2)}=c_{n, k, j, i}^{(2), \mathrm{re}}+\mathrm{i} c_{n, k, j, i}^{(2), \text { im }}$. For the real part of $\mathcal{L}^{-1}\left(R_{n, k}^{(2)}\right)$ we get

$$
\begin{aligned}
\mathcal{L}_{\mathrm{re}}^{-1}\left(R_{n, k}^{(2)}\right)(t)= & (-1)^{n k} \delta(t-2 k) H(t-2 k) \\
& +\sum_{i=1}^{n} \sum_{j=1}^{k} c_{n, k, j, i}^{(2), \mathrm{re}} H(t-2 k)(t-2 k)^{j-1} e^{-\alpha_{i}^{\mathrm{re}}(t-2 k)} \cos \left(-\alpha_{i}^{\mathrm{im}}(t-2 k)\right) \\
& -\sum_{i=1}^{n} \sum_{j=1}^{k} c_{n, k, j, i}^{(2), \mathrm{im}} H(t-2 k)(t-2 k)^{j-1} e^{-\alpha_{i}^{\mathrm{re}}(t-2 k)} \sin \left(-\alpha_{i}^{\mathrm{im}}(t-2 k)\right) .
\end{aligned}
$$

For the inverse Laplace transform of $R_{n, k}^{(3)}$ we use the shift property again and get

$$
\begin{aligned}
\mathcal{L}^{-1}\left(R_{n, k}^{(3)}\right)(t) & =\mathcal{L}^{-1}\left(\frac{\tilde{\theta}_{2 n-1}(s) \theta_{n}(s)^{k-1}}{\theta_{n}(-s)^{k+1}} e^{-2 k s}\right)(t) \\
& =H(t-2 k) \mathcal{L}^{-1}\left(\frac{\tilde{\theta}_{2 n-1}(s) \theta_{n}(s)^{k-1}}{\theta_{n}(-s)^{k+1}}\right)(t-2 k) \\
& =H(t-2 k)\left[\sum_{i=1}^{n} \sum_{j=1}^{k} c_{n, k, j, i}^{(3)}(t-2 k)^{j} e^{-\alpha_{i}(t-2 k)}\right] .
\end{aligned}
$$

The real part of $\mathcal{L}^{-1}\left(R_{n, k}^{(2)}\right)$ can therefore be written as

$$
\begin{aligned}
\mathcal{L}_{\mathrm{re}}^{-1}\left(R_{n, k}^{(3)}\right)(t)= & \sum_{i=1}^{n} \sum_{j=1}^{k} c_{n, k, j, i}^{(3), \mathrm{re}} H(t-2 k)(t-2 k)^{j} e^{-\alpha_{i}^{\mathrm{re}}(t-2 k)} \cos \left(-\alpha_{i}^{\mathrm{im}}(t-2 k)\right) \\
& -\sum_{i=1}^{n} \sum_{j=1}^{k} c_{n, k, j, i}^{(3), \mathrm{im}} H(t-2 k)(t-2 k)^{j} e^{-\alpha_{i}^{\mathrm{re}}(t-2 k)} \sin \left(-\alpha_{i}^{\mathrm{im}}(t-2 k)\right) .
\end{aligned}
$$




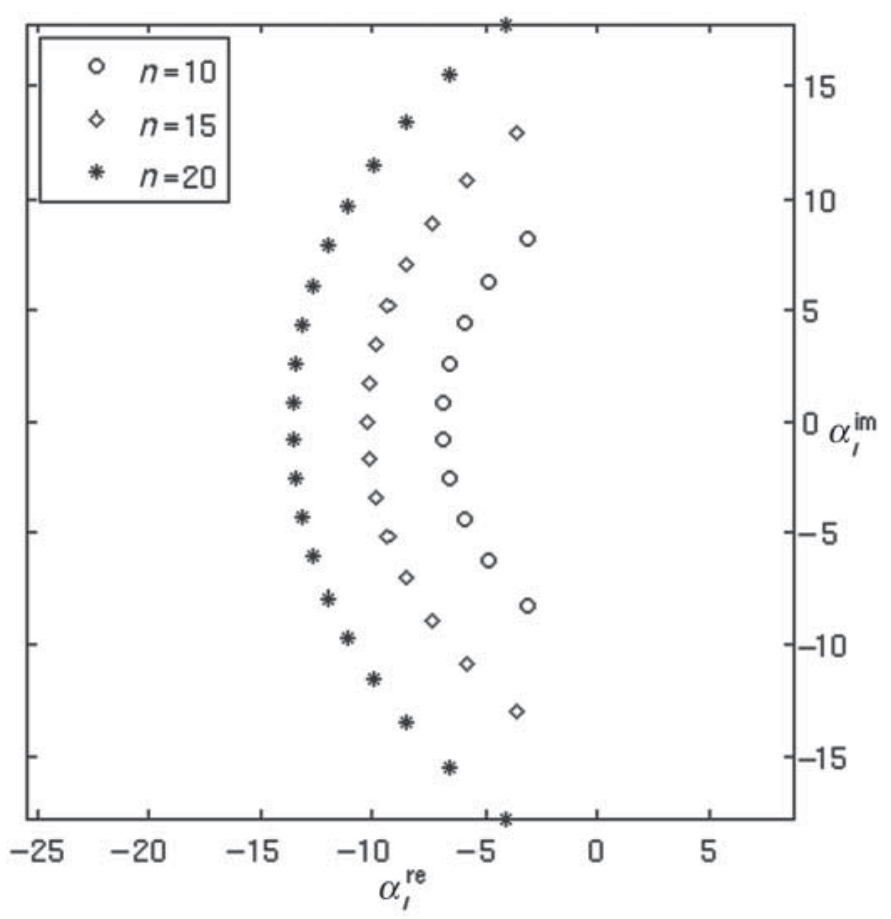

FIG. 2. Complex zeros of $\theta_{10}(s), \theta_{15}(s)$ and $\theta_{20}(s)$.

With these formulas for $\mathcal{L}_{\mathrm{re}}^{-1}\left(R_{n}^{(1)}\right), \mathcal{L}_{\mathrm{re}}^{-1}\left(R_{n, k}^{(2)}\right)$ and $\mathcal{L}_{\mathrm{re}}^{-1}\left(R_{n, k}^{(3)}\right)$ it is now possible to invert the remaining term in (4.6). Inserting this in (4.4) leads to explicit formulas for the exact solution $\phi(t)$.

REMARK 4.5 Note that the complex zeros of $\theta_{n}(s)$ are located in left half plane of $\mathbb{R}^{2}$, that is, $-\alpha_{i}^{\text {re }}>0$ for any $i$ and $n$ (cf. Fig. 2, also Ismail, 2009). The behaviour of the solution $\phi(t)$ of (4.4) is typically oscillatory and bounded for large time, while the representations which we will derive contain exponentially increasing functions (which cancel each other). Hence, for larger order $n \geqslant 5$, these formulas are useful only for small times because of round-off errors- the use of computer programs such as Mathematica and Maple, with adaptive or even exact working arithmetic might reduce this problem substantially. Since our representations of $\phi$ are explicit they can be a starting point, for example, for analysing the regularity of the solution depending on the compatibility of the right-hand side $g(t)$ at $t=0$. In addition, their implementation is straightforward so that they can be used to generate reference solutions, for example, for studying the convergence of a new discretization method for the convolution equation (4.3) and thus of the full problem (2.3) — of course the problem of round-off errors has to be taken into account by restricting to sufficiently small time intervals.

\subsection{The case $n=0$}

For $n=0$ the eigenfunctions in (4.2) are constant. We are therefore in the case where

$$
g(x, t):=2 \sqrt{\pi} Y_{0}^{0} g(t)=g(t)
$$


is purely time dependent. This case has already been treated in Banjai \& Sauter (2008) and an explicit representation of $\phi(t)$ in (4.4) was given for $t \in[0,2[$. We generalize this to $t \geqslant 0$. Therefore, note that the associated eigenvalue in this case is given by

$$
\lambda_{0}(s)=\frac{1-e^{-2 s}}{2 s},
$$

and from the above computations we can see that

$$
\mathcal{L}\left(\frac{1}{\lambda_{0}}\right)(t)=2 \delta^{\prime}(t)+2 \partial_{t}\left(\sum_{k=1}^{\infty} \delta(t-2 k) H(t-2 k)\right) .
$$

Therefore, the exact solution in this simple case is given by

$$
\begin{aligned}
\phi(t) & =\int_{0}^{t} g(t-\tau)\left[2 \delta^{\prime}(\tau)+2 \partial_{\tau}\left(\sum_{k=1}^{\infty} \delta(\tau-2 k) H(\tau-2 k)\right)\right] \mathrm{d} \tau \\
& =2 g^{\prime}(t)+2 \sum_{k=1}^{\infty} \int_{0}^{t} g(t-\tau) \partial_{t}(\delta(\tau-2 k) H(\tau-2 k)) \mathrm{d} \tau \\
& =2 g^{\prime}(t)+2 \sum_{k=1}^{\infty} g^{\prime}(t-2 k) \\
& =2 \sum_{k=0}^{\lfloor t / 2\rfloor} g^{\prime}(t-2 k)
\end{aligned}
$$

due to the causality of $g$. Figure 3 shows a typical behaviour of $\phi(t)$. Note the oscillatory, nondecaying shape of the solution for larger times $t$. This is due to the fact that in indirect methods $\phi(t)$ is the trace difference of the solution of the exterior and the solution of the interior wave equation. The latter is determined by the many reflections inside the sphere and therefore causes the oscillations in the solution.

A closer look at Fig. 3 suggests that $\phi(t)$ becomes a very regular function for large times. Indeed it can be shown that $\phi(t)$ tends to a periodic function for sufficiently fast-decaying right-hand sides $g(t)$. In order to see that, we set

$$
t=2 l+\tau, \quad \tau \in\left[0,2\left[, \quad l \in \mathbb{N}_{0},\right.\right.
$$

and get

$$
\phi(2 l+\tau)=2 \sum_{k=0}^{l} g^{\prime}(2 k+\tau)
$$

Suppose that $g(t)$ satisfies

$$
\begin{aligned}
& g(0)=g^{\prime}(0)=0, \\
& \left|g^{\prime}(t)\right| \leqslant C t^{-\alpha},
\end{aligned}
$$

for $t>0$ with $\alpha>1$ and a positive constant $C$. With these assumptions, the following lemma holds. 

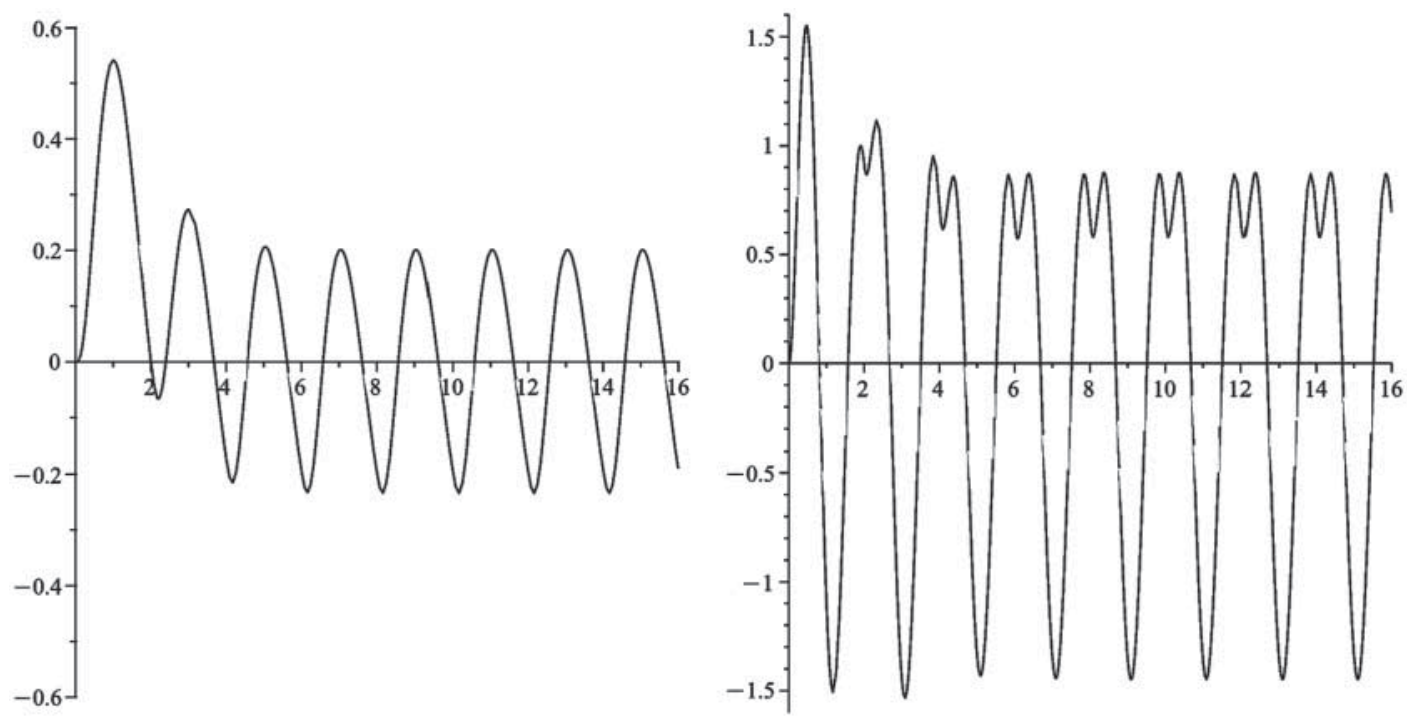

Fig. 3. Exact solution $\phi(t)$ of (4.3) with $n=0$ for $g(t)=t^{4} e^{-2 t}$ (left) and $g(t)=\sin (2 t)^{2} t e^{-t}$ (right).

Lemma 4.6 Let (4.12) and (4.13) be satisfied. Then, the sequence of functions $\{\phi(2 l+\tau)\}_{l \in \mathbb{N}_{0}}$ converges uniformly to a function $f(\tau):[0,2[\rightarrow \mathbb{R}$.

Proof. Let $\varepsilon>0$. Since $\alpha>1$, we find $N \in \mathbb{N}$ such that

$$
\sum_{k=l+1}^{m}(2 k+2)^{-\alpha}<\frac{\varepsilon}{2 C}
$$

for all $m>l>N$. Thus,

$$
\begin{aligned}
|\phi(2 m+\tau)-\phi(2 l+\tau)| & \leqslant 2 \sum_{k=l+1}^{m}\left|g^{\prime}(2 k+\tau)\right| \leqslant 2 C \sum_{k=l+1}^{m}(2 k+\tau)^{-\alpha} \\
& \leqslant 2 C \sum_{k=l+1}^{m}(2 k+2)^{-\alpha} \leqslant \varepsilon
\end{aligned}
$$

for all $m>l>N$ and therefore the convergence is uniform.

Corollary 4.7 The limit function $f(\tau)$ is continuous and satisfies

$$
f(0)=\lim _{\tau \rightarrow 2} f(\tau)
$$

The solution of the scattering problem therefore tends to a periodic function for large times for every right-hand side satisfying (4.12) and (4.13). 
Proof. We see that $f(\tau)$ is continuous since the uniform limit of continuous functions is continuous. Furthermore,

$$
\lim _{\tau \rightarrow 2} f(\tau)=\lim _{\tau \rightarrow 2} \lim _{n \rightarrow \infty} \phi(2 n+\tau)=\lim _{n \rightarrow \infty} \lim _{\tau \rightarrow 2} \phi(2 n+\tau)=\lim _{n \rightarrow \infty} \phi(2 n+2)=f(0),
$$

again due to the continuity of $\phi$.

Let us suppose now that $g(t)$ is of the form

$$
g(t)=v(t) e^{-\alpha t} \quad \text { with } v(t)=t^{2} p(t),
$$

where $p \in \mathbb{P}_{q}$ is a polynomial of degree $q$. In this case we can compute the limit function $f(\tau)$ explicitly. Let the constant $c_{m}$ be defined as

$$
c_{m}:=\frac{v^{(m+1)}(0)-\alpha v^{(m)}(0)}{m !} .
$$

Expanding $v(t)$ and $v^{\prime}(t)$ about 0 leads to

$$
\begin{aligned}
& \phi(2 l+\tau)=2 \sum_{k=0}^{l}\left[v^{\prime}(2 k+\tau)-\alpha v(2 k+\tau)\right] e^{-\alpha \tau-2 \alpha k} \\
& =2 \sum_{k=0}^{l}\left[\sum_{m=1}^{q} c_{m}(2 k+\tau)^{m}\right] e^{-\alpha \tau-2 \alpha k} \\
& =2 e^{-\alpha \tau} \sum_{m=1}^{q} \sum_{k=0}^{l} c_{m}(2 k+\tau)^{m} e^{-2 \alpha k} \\
& =2 e^{-\alpha \tau} \sum_{m=1}^{q} \sum_{k=0}^{l} c_{m}\left(\sum_{j=0}^{m}\left(\begin{array}{c}
m \\
j
\end{array}\right) \tau^{m-j}(2 k)^{j}\right) e^{-2 \alpha k} \\
& =2 e^{-\alpha \tau} \sum_{m=1}^{q} \sum_{j=0}^{m} 2^{j}\left(\begin{array}{c}
m \\
j
\end{array}\right) c_{m} \tau^{m-j} \underbrace{\sum_{k=0}^{l} k^{j} e^{-2 \alpha k}}_{=: R_{l, j, \alpha}} .
\end{aligned}
$$

We are interested in $\phi(t)$ for large times $t$. Therefore, we need an expression for $R_{l, j, \alpha}$ when $l$ tends to infinity.

Lemma 4.8 Let $j \in \mathbb{N}$ and $\alpha \in \mathbb{R}_{>0}$ be fixed. Then

$$
\sum_{k=0}^{\infty} k^{j} e^{-2 \alpha k}=\sum_{m=0}^{j} \sum_{q=0}^{m} \frac{\left.(-1)^{m-q} q^{j(} \begin{array}{c}
j+1 \\
m-q
\end{array}\right) e^{2 \alpha(j-m+1)}}{\left(e^{2 \alpha}-1\right)^{j+1}}
$$


Proof. Since we want to compute $\lim _{l \rightarrow \infty} R_{l, j, \alpha}$, we assume that $l \geqslant j$. We get

$$
\begin{aligned}
{\left[\sum_{k=0}^{l} k^{j} e^{-2 \alpha k}\right]\left(e^{2 \alpha}-1\right)^{j+1}=} & \sum_{k=0}^{l} \sum_{q=0}^{j+1}(-1)^{j-q+1} k^{j}\left(\begin{array}{c}
j+1 \\
q
\end{array}\right) e^{-2 \alpha(k-q)} \\
= & \sum_{m=-(j+1)}^{-1} \sum_{q=-m}^{j+1}(-1)^{j+1-q}(q+m)^{j}\left(\begin{array}{c}
j+1 \\
q
\end{array}\right) e^{-2 \alpha m} \\
& +\sum_{m=0}^{l-j-1} \sum_{q=0}^{j+1}(-1)^{j+1-q}(q+m)^{j}\left(\begin{array}{c}
j+1 \\
q
\end{array}\right) e^{-2 \alpha m} \\
& +\sum_{m=l-j}^{l} \sum_{q=0}^{l-m}(-1)^{j+1-q}(q+m)^{j}\left(\begin{array}{c}
j+1 \\
q
\end{array}\right) e^{-2 \alpha m} .
\end{aligned}
$$

The second double sum in the last term is zero since, for any polynomial $p$ of degree less than $j$, the equation

$$
\sum_{q=0}^{j}(-1)^{q} p(q)\left(\begin{array}{l}
j \\
q
\end{array}\right)=0
$$

holds. Therefore,

$$
\begin{aligned}
{\left[\sum_{k=0}^{l} k^{j} e^{-2 \alpha k}\right]\left(e^{2 \alpha}-1\right)^{j+1}=} & \sum_{m=-(j+1)}^{-1} \sum_{q=-m}^{j+1}(-1)^{j+1-q}(q+m)^{j}\left(\begin{array}{c}
j+1 \\
q
\end{array}\right) e^{-2 \alpha m} \\
& +\sum_{m=-j}^{0} \sum_{q=0}^{-m}(-1)^{j+1-q}(q+l+m)^{j}\left(\begin{array}{c}
j+1 \\
q
\end{array}\right) e^{-2 \alpha(l+m)} .
\end{aligned}
$$

Now, we can pass to the limit for $l \rightarrow \infty$ where the second double sum vanishes. After a reordering of the terms we get

$$
\left[\sum_{k=0}^{\infty} k^{j} e^{-2 \alpha k}\right]\left(e^{2 \alpha}-1\right)^{j+1}=\sum_{m=0}^{j} \sum_{q=0}^{m}(-1)^{m-q} q^{j}\left(\begin{array}{c}
j+1 \\
m-q
\end{array}\right) e^{2 \alpha(j-m+1)} .
$$

Dividing by $\left(e^{2 \alpha}-1\right)^{j+1}$ leads to the desired result.

If we assume a right-hand side of the form (4.14) we get, by Lemma 4.8, that

$$
\phi(2 l+\tau) \underset{l \rightarrow \infty}{\longrightarrow} f(\tau) \tau \in[0,2[,
$$

where $f$ is given by

$$
f(\tau)=2 e^{-\alpha \tau} \sum_{m=1}^{q} \sum_{j=0}^{m} \tilde{c}_{m, j, \alpha} \tau^{m-j}
$$


and

$$
\tilde{c}_{m, j, \alpha}=c_{m} \sum_{k=0}^{j} \sum_{q=0}^{k}(-1)^{k-q}(2 q)^{j}\left(\begin{array}{c}
m \\
j
\end{array}\right)\left(\begin{array}{l}
j+1 \\
k-q
\end{array}\right) e^{2 \alpha(j-k+1)}\left(e^{2 \alpha}-1\right)^{-j-1} .
$$

With Lemma 4.8 it is also possible to show that the convergence in (4.15) is exponentially fast in $l$ up to a polynomial factor if $g(t)$ is decaying exponentially.

Lemma 4.9 Suppose that $g(t)$ is of the form

$$
g(t)=v(t) e^{-\alpha t}
$$

with $\alpha>0$, where $v(t)$ is a continuous function satisfying

$$
\begin{aligned}
& v(0)=v^{\prime}(0)=0, \\
& |v(t)| \leqslant C_{1} t^{p_{1}}, \\
& \left|v^{\prime}(t)\right| \leqslant C_{2} t^{p_{2}},
\end{aligned}
$$

for some $p_{1}, p_{2} \in \mathbb{N}$ and positive constants $C_{1}$ and $C_{2}$. For $l \geqslant \max \left\{p_{1}, p_{2}\right\}$ we have

$$
\sup _{\tau \in[0,2[}|f(\tau)-\phi(2 l+\tau)| \leqslant p(l+1) e^{-2 \alpha(l+1)},
$$

where $p$ is a polynomial of degree $\max \left\{p_{1}, p_{2}\right\}$ and $f$ is as in Lemma 4.6.

Proof. From the proof of Lemma 4.8 it follows

$$
\sum_{k=l+1}^{\infty} k^{j} e^{-2 \alpha k} \leqslant l^{j} e^{-2 \alpha l} \underbrace{\sum_{m=-j}^{0} \sum_{i=0}^{-m} \frac{\left(\begin{array}{c}
j+1 \\
i
\end{array}\right) e^{-2 \alpha m}}{\left(e^{2 \alpha}-1\right)^{j+1}}}_{=: c_{\alpha, j}}
$$

for $l \geqslant j$. Then, we get

$$
\begin{aligned}
|f(\tau)-\phi(2 l+\tau)| & \leqslant 2 \sum_{k=l+1}^{\infty}\left|g^{\prime}(2 k+\tau)\right| \\
& =2 \sum_{k=l+1}^{\infty}\left|u^{\prime}(2 k+\tau)-\alpha u(2 k+\tau)\right| e^{-\alpha \tau-2 \alpha k} \\
& \leqslant 2 e^{-\alpha \tau}\left(\sum_{k=l+1}^{\infty}\left|u^{\prime}(2 k+\tau)\right| e^{-2 \alpha k}+\sum_{k=l+1}^{\infty} \alpha|u(2 k+\tau)| e^{-2 \alpha k}\right)
\end{aligned}
$$




$$
\begin{aligned}
& \leqslant 2 e^{-\alpha \tau}\left(\sum_{k=l+1}^{\infty} C_{2}(2 k+\tau)^{p_{2}} e^{-2 \alpha k}+\sum_{k=l+1}^{\infty} \alpha C_{1}(2 k+\tau)^{p_{1}} e^{-2 \alpha k}\right) \\
& \leqslant C_{2} 2^{p_{2}+1} \sum_{k=l+1}^{\infty}(k+1)^{p_{2}} e^{-2 \alpha k}+\alpha C_{1} 2^{p_{1}+1} \sum_{k=l+1}^{\infty}(k+1)^{p_{1}} e^{-2 \alpha k} \\
& =C_{2} 2^{p_{2}+1} e^{2 \alpha} \sum_{k=l+2}^{\infty} k^{p_{2}} e^{-2 \alpha k}+\alpha C_{1} 2^{p_{1}+1} e^{2 \alpha} \sum_{k=l+2}^{\infty} k^{p_{1}} e^{-2 \alpha k} \\
& \leqslant\left[C_{2} 2^{p_{2}+1} c_{\alpha, p_{2}}(l+1)^{p_{2}}+\alpha C_{1} 2^{p_{1}+1} c_{\alpha, p_{1}}(l+1)^{p_{1}}\right] e^{-2 \alpha(l+1)}
\end{aligned}
$$

for arbitrary $\tau \in[0,2[$.

\subsection{The case $n=1$}

In the case of linear eigenfunctions in (4.2) the representation of the solution $\phi(t)$ becomes more complicated than in the previous case. For $n=1$ the eigenvalue is given by

$$
\lambda_{1}(s)=\frac{-\theta_{1}(-s) \theta_{1}(s)+\theta_{1}^{2}(s) e^{-2 s}}{2 s^{3}}
$$

where

$$
\theta_{1}(s)=s+1 \text {. }
$$

Note that $\lambda_{1}$ has one real zero, namely $\alpha_{1}=-1$. With the above computations we get

$$
\begin{aligned}
\mathcal{L}^{-1} & \left(\frac{\tilde{\theta}_{0}(s)-\theta_{1}(s)^{2} e^{-2 s}}{\theta_{1}(-s) \theta_{1}(s)-\theta_{1}(s)^{2} e^{-2 s}}\right)(t) \\
& \stackrel{(4.7)}{=} \mathcal{L}^{-1}\left(R_{1}^{(1)}\right)(t)+\sum_{k=1}^{\infty}\left(-\mathcal{L}^{-1}\left(R_{1, k}^{(2)}\right)(t)+\mathcal{L}^{-1}\left(R_{1, k}^{(3)}\right)(t)\right) \\
& \stackrel{(4.8)}{=}-\sinh (t) \stackrel{(4.9)}{-} \sum_{k=1}^{\infty}(-1)^{k} \delta(t-2 k) H(t-2 k)-\sum_{k=1}^{\infty} \sum_{j=1}^{k} c_{1, k, j, 1}^{(2), \mathrm{re}} H(t-2 k)(t-2 k)^{j-1} e^{t-2 k} \\
& \quad+\sum_{k=1}^{(4.10)} \sum_{j=1}^{k} c_{1, k, j, 1}^{(3), \mathrm{re}} H(t-2 k)(t-2 k)^{j} e^{t-2 k} \\
= & -\sinh (t)+\sum_{k=1}^{\infty}(-1)^{k+1} \delta(t-2 k) H(t-2 k) \\
& +\sum_{k=1}^{\infty}\left[\sum_{j=1}^{k}\left(c_{k, j}^{(2)}+c_{k, j}^{(3)} t-c_{k, j}^{(3)} 2 k\right)(t-2 k)^{j-1} e^{t-2 k}\right] H(t-2 k),
\end{aligned}
$$

where

$$
c_{k, j}^{(2)}:=c_{1, k, j, 1}^{(2), \mathrm{re}} \quad \text { and } \quad c_{k, j}^{(3)}:=c_{1, k, j, 1}^{(3), \mathrm{re}} \text {. }
$$


With the formulas in Erdélyi et al. (1954, Section 5.2) we obtain the following explicit expressions for these constants:

$$
c_{k, j}^{(2)}=(-1)^{k+1} \sum_{m=0}^{j-1} \frac{\left(1-(-1)^{j-m}\right) k !}{(j-1) ! m !(k-j) !(j-m) !}
$$

and

$$
c_{k, j}^{(3)}=(-1)^{k+1} \frac{2^{j-1}(k-1) !}{(j-1) ! j !(k-j) !},
$$

where we used

$$
\frac{(1+s)^{k}}{(1-s)^{k}}=(-1)^{k}+\frac{\sum_{i=0}^{k-1}\left(\begin{array}{l}
k \\
i
\end{array}\right)(-1)^{k}\left(1-(-1)^{k-i}\right) s^{i}}{(s-1)^{k}},
$$

in order to compute $c_{k, j}^{(2)}$. With (4.6) and (4.4) we therefore get for the solution

$$
\begin{aligned}
\phi(t)= & \int_{0}^{t} g(t-\tau) \mathcal{L}^{-1}\left(\frac{1}{\lambda_{1}}\right)(\tau) \mathrm{d} \tau \\
= & 2 g^{\prime}(t)-2 \int_{0}^{t}\left(-\sinh (\tau)+\sum_{k=1}^{\infty}(-1)^{k+1} \delta(\tau-2 k) H(\tau-2 k)\right. \\
& \left.+\sum_{k=1}^{\infty} \sum_{j=1}^{k}\left(c_{k, j}^{(2)}+c_{k, j}^{(3)} \tau-c_{k, j}^{(3)} 2 k\right)(\tau-2 k)^{j-1} e^{\tau-2 k} H(\tau-2 k)\right) g^{\prime}(t-\tau) \mathrm{d} \tau \\
= & 2 g^{\prime}(t)+2 \sum_{k=1}^{\lfloor t / 2\rfloor}(-1)^{k} g^{\prime}(t-2 k)+2 \int_{0}^{t} \sinh (\tau) g^{\prime}(t-\tau) \mathrm{d} \tau \\
& -2 \sum_{k=1}^{\infty} \sum_{j=1}^{k} \int_{0}^{t}\left(c_{k, j}^{(2)}+c_{k, j}^{(3)} \tau-c_{k, j}^{(3)} 2 k\right)(\tau-2 k)^{j-1} e^{\tau-2 k} H(\tau-2 k) g^{\prime}(t-\tau) \mathrm{d} \tau \\
= & 2 \sum_{k=0}^{\lfloor t / 2\rfloor}(-1)^{k} g^{\prime}(t-2 k)+2 \int_{0}^{t} \sinh (\tau) g^{\prime}(t-\tau) \mathrm{d} \tau \\
& -2 \sum_{k=1}^{\lfloor t / 2\rfloor} \sum_{j=1}^{k} \int_{2 k}^{t}\left(c_{k, j}^{(2)}+c_{k, j}^{(3)} \tau-c_{k, j}^{(3)} 2 k\right)(\tau-2 k)^{j-1} e^{\tau-2 k} g^{\prime}(t-\tau) \mathrm{d} \tau .
\end{aligned}
$$

Figure 4 shows solutions for different right-hand sides $g(t)$. As for the case $n=0$ we have oscillatory behaviour for larger times $t$ which is again due to the shape of the solution of the interior wave problem. Similar properties of these solutions reported before could not be observed; that is, in general $\phi(t)$ does not seem to adopt a simple periodic pattern as time evolves.

\section{Numerical experiments}

In this section we present the results of numerical experiments. We first want to verify the sharpness of Lemma 4.9 for different right-hand sides $g$. Let 

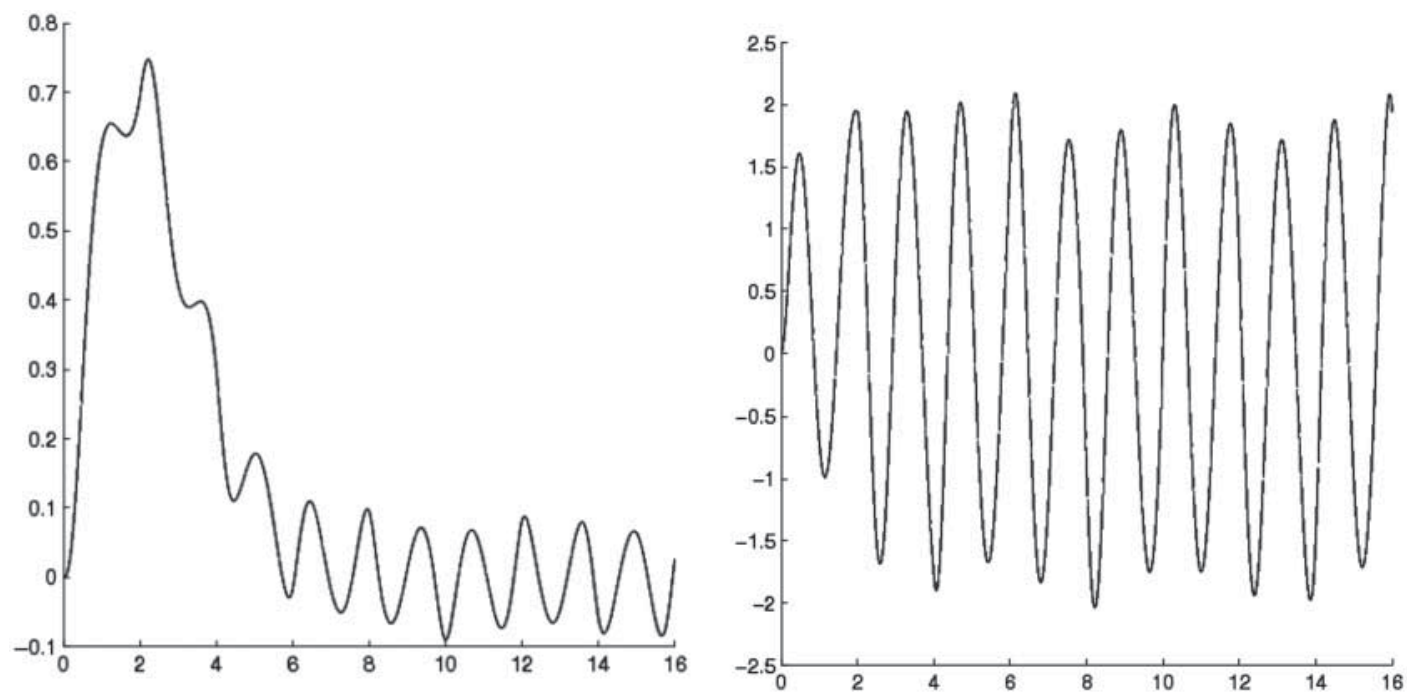

FIG. 4. Exact solution $\phi(t)$ of (4.3) with $n=1$ for $g(t)=t^{4} e^{-2 t}$ (left) and $g(t)=\sin (2 t)^{2} t e^{-t}$ (right).

$$
\begin{aligned}
& g_{1}(t)=t^{4} e^{-2 t}, \quad g_{2}(t)=t^{2} e^{-2 t}, \\
& g_{3}(t)=t \sin (t) e^{-t}, \quad g_{4}(t)=\frac{1}{4} t \sin (5 t) e^{-t},
\end{aligned}
$$

and denote by $\phi_{j}, j \in\{1,2,3,4\}$ the corresponding solutions of the boundary integral equation. Let $f_{j}:[0,2[\rightarrow \mathbb{R}, j \in\{1,2,3,4\}$ be the limit functions corresponding to these solutions as in Lemma 4.6. We define the errors

$$
\operatorname{err}_{j}(l):=\left\|f_{j}(\cdot)-\phi_{j}(2 l-\cdot)\right\|_{L^{\infty}([0,2])}, \quad j \in\{1,2,3,4\}
$$

and illustrate the convergence in Figs 5 and 6. As predicted by Lemma 4.9 the solutions converge in all cases exponentially fast against the corresponding limit functions due to the exponential decay of the right-hand sides. Since the degree of the increasing polynomial factor in $g_{1}$ is higher than in $g_{2}$ the error err ${ }_{1}$ decays slower than $\operatorname{err}_{2}$ by a polynomial factor (cf. Fig. 6). The cases $g_{3}$ and $g_{4}$ indicate that more oscillatory right-hand sides (and therefore more oscillatory solutions) do not lead to a slower convergence rate if the decay behaviour of these functions is the same.

We now turn our attention to the approximation of $\phi$ in (2.3) by a Galerkin method using the basis functions $b_{i}$ defined in Section 3 (see Sauter \& Veit, 2013 for details) in time and piecewise linear basis functions in space. We apply Algorithm 1 and compute approximations of the form

$$
\phi_{\text {Galerkin }}=\sum_{i=1}^{L} \sum_{j=1}^{M} \alpha_{i}^{j} \varphi_{j}(x) b_{i}(t), \quad \alpha_{i}^{j} \in \mathbb{R},
$$

where the number of basis functions in time, $L$, depends on the number of time steps and the degree $p$ of the local polynomial approximation spaces used. We measure the resulting error, $\phi_{\text {exact }}-\phi_{\text {Galerkin }}$, in 


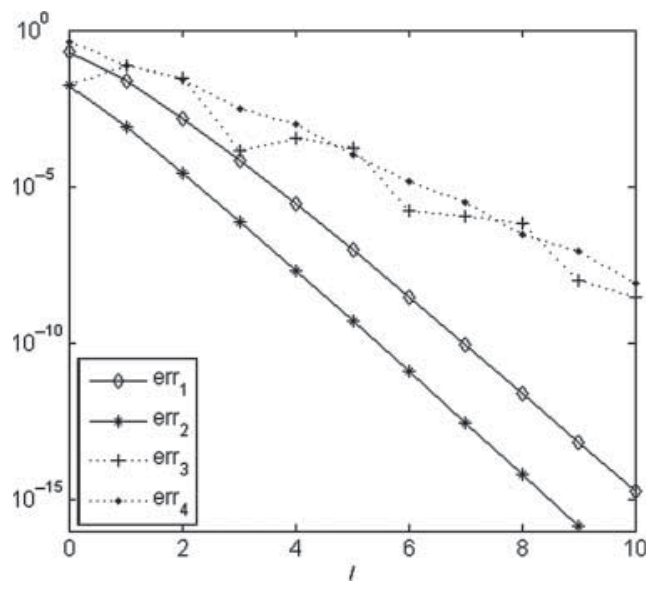

FIG. 5. $\operatorname{err}_{j}(l)$ for $j=1,2,3,4$.

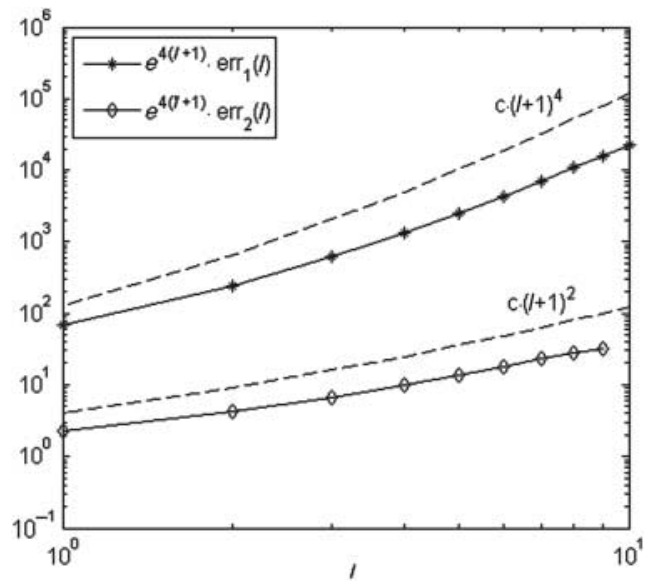

FIG. 6. Log-log scale plot of $e^{4(l+1)} \cdot \operatorname{err}_{j}(l)$ for $j=1,2$.

the $L^{2}\left((0, T), L^{2}(\Gamma)\right)$ norm and denote by

$$
\operatorname{err}_{\text {rel }}:=\frac{\left\|\phi_{\text {exact }}-\phi_{\text {Galerkin }}\right\|_{L^{2}\left((0, T), L^{2}(\Gamma)\right)}}{\left\|\phi_{\text {exact }}\right\|_{L^{2}\left((0, T), L^{2}(\Gamma)\right)}}
$$

the corresponding relative error.

In the following we consider the case of a spherical scatterer, that is, $\Gamma=\mathbb{S}^{2}$. In the first experiment we assume that the right-hand side is given by $g(x, t)=t^{4} e^{-6 t} Y_{n}^{0}, n=2,3$. We showed above that the exact solution in this case is of the form $\phi(x, t)=\phi(t) Y_{n}^{0}, n=2,3$. Figure 7 shows the time part of the solutions, $\phi(t)$, for these two problems. They were computed using the formulas derived in the 


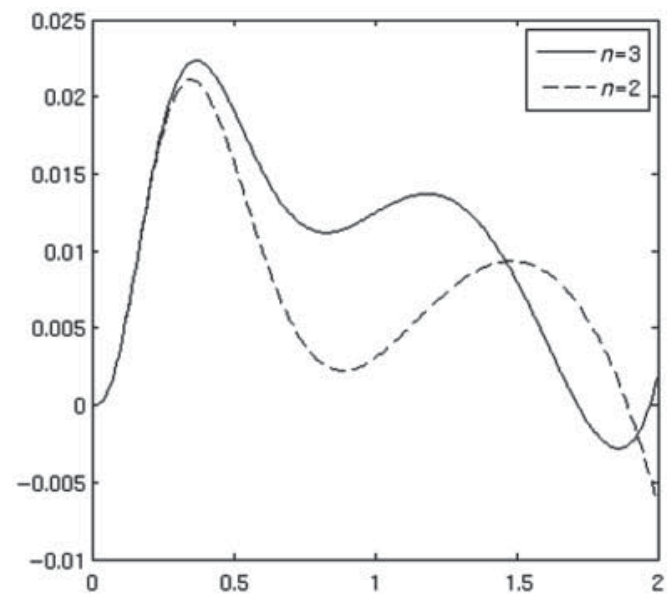

FIG. 7. Time part of the exact solution for $g(t, x)=t^{4} e^{-6 t} Y_{n}^{0}$ and $n=2,3$.

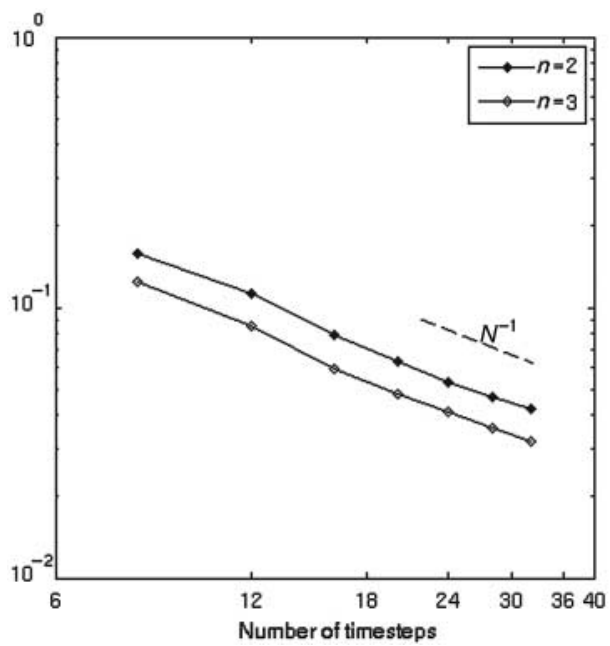

FIG. 8. Relative error $\operatorname{err}_{\text {rel }}$ for $T=2, g(x, t)=t^{4} e^{-6 t} Y_{n}^{0}$ and $n=2,3$, where local polynomial approximation spaces of degree $p=0$ were used.

last section. Figure 8 shows the error that results from approximating these solutions by functions of the form (5.1). In this case we computed approximations in the time interval $[0,2]$ using equidistant time steps and local polynomial approximation spaces of degree $p=0$; that is, the approximations in time are simply linear combinations of the partition-of-unity functions defined in Section 3. In space we used an approximation of the sphere using 616 flat triangles and piecewise linear basis functions. In both cases a convergence order of $N^{-1}$ is obtained, where $N$ is the number of time steps.

In a second experiment we again set $\Gamma=\mathbb{S}^{2}$ and assume the right-hand side $g(x, t)=\sin (t)^{4} e^{-0.5 t} Y_{n}^{0}$ for $n=2,3$. We consider the time interval $[0,2]$, fix the number of time steps at 25 and approximate in 


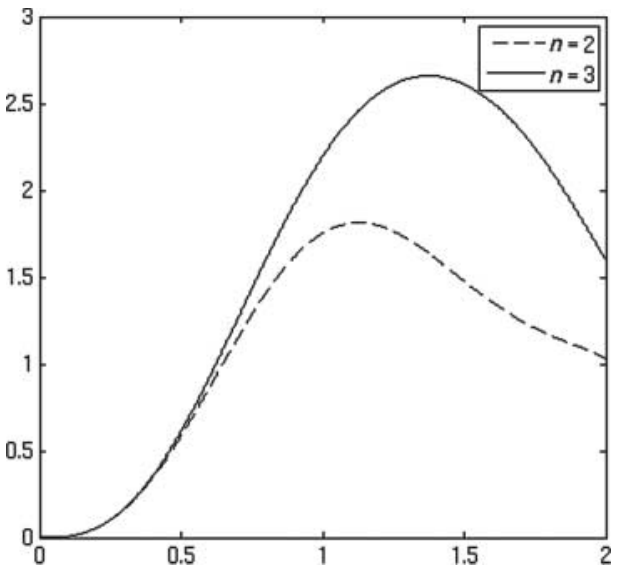

FIG. 9. Time part of the exact solution for $g(t, x)=\sin (t)^{4} e^{-0.5 t} Y_{n}^{0}$ and $n=2,3$.

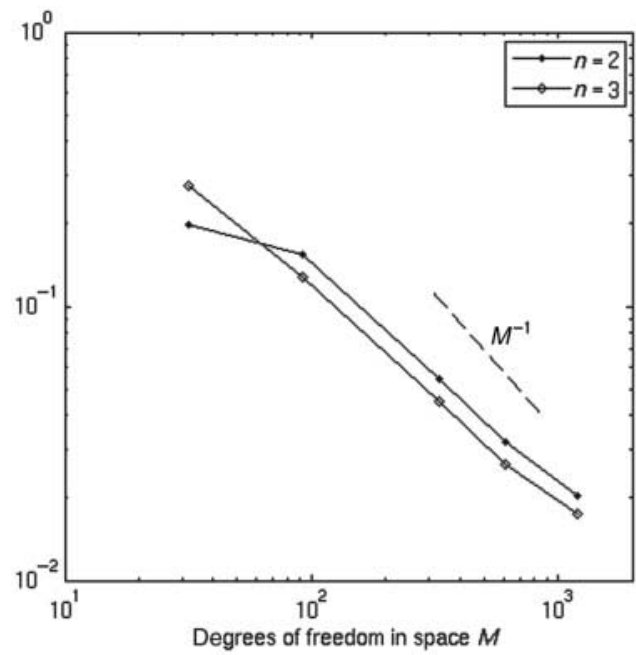

FIG. 10. Relative error $\operatorname{err}_{\text {rel }}$ for $T=2, g(x, t)=\sin (t)^{4} e^{-0.5 t} Y_{n}^{0}$ with $n=2,3$ and piecewise constant approximation in space.

time with local polynomial approximation spaces of degree $p=1$. In space we approximate the solution with piecewise constant functions defined on a triangulation of the sphere with $M$ flat triangles. Figure 9 shows the time part of the solutions for these problems. Figure 10 shows the $L^{2}\left((0, T), L^{2}(\Gamma)\right)$ error with respect to the number of triangles $M$. Although the theory predicts an asymptotic convergence order of $M^{-1}$, the numerical experiment shows a slightly slower decay. This is due to additional errors arising from the surface approximation of $\Gamma$ and the approximate evaluation of the $L^{2}\left((0, T), L^{2}(\Gamma)\right)$ norm using quadrature. 


\section{Conclusion}

We considered retarded boundary integral formulations of the three-dimensional wave equation in unbounded domains. We formulated an algorithm for the space-time Galerkin discretization using the smooth and compactly supported temporal basis function developed in Sauter \& Veit (2013). In order to test these basis functions numerically we derived explicit representations of the exact solutions of the integral equations in the case where the scatterer is the unit ball in $\mathbb{R}^{3}$ and special Dirichlet boundary conditions have to be satisfied. Furthermore, we showed some analytic properties of these solutions in the case where the right-hand side is purely time dependent.

The implementation of the obtained formulas is simple since only the right-hand side, its first derivative with respect to time and, depending on $n$, numerical quadrature is needed for the numerical evaluation. They can therefore serve as reference solutions in order to test numerical approximation schemes.

\section{Funding}

The second author gratefully acknowledges the support given by the Swiss National Science Foundation, No. PDFMP2_127437/1.

\section{REFERENCES}

Abramowitz, M. \& Stegun, I. (1972) Handbook of Mathematical Functions. Applied Mathematics Series, vol. 55. National Bureau of Standards, U.S. Department of Commerce. Abramowitz, Stegun: Washington.

BAMBERGER, A. \& DuONG, T. H. (1986) Formulation variationnelle espace-temps pur le calcul par potientiel retardé de la diffraction d'une Onde Acoustique. Math. Methods Appl. Sci., 8, 405-435.

BANJAI, L. (2010) Multistep and multistage convolution quadrature for the wave equation: algorithms and experiments. SIAM J. Sci. Comput., 32, 2964-2994.

Banjai, L., Melenk, J. \& Lubich, C. (2011) Runge-Kutta convolution quadrature for operators arising in wave propagation. Numer. Math., 119, 1-20.

BANJAI, L. \& SAUTER, S. (2008) Rapid solution of the wave equation in unbounded domains. SIAM J. Numer. Anal., 47, 227-249.

BANJAI, L. \& SchanZ, M. (2012) Wave propagation problems treated with convolution quadrature and BEM. Fast Boundary Element Methods in Engineering and Industrial Applications (U. Langer, M. Schanz, O. Steinbach \& W. L. Wendland eds), Lecture Notes in Applied and Computational Mechanics, vol. 63. Berlin: Springer, pp. $145-184$.

Birgisson, B., Siebrits, E. \& Peirce, A. (1999) Elastodynamic direct boundary element methods with enhanced numerical stability properties. Internat. J. Numer. Methods Engrg., 46, 871-888.

Bluck, M. \& Walker, S. (1996) Analysis of three dimensional transient acoustic wave propagation using the boundary integral equation method. Internat. J. Numer. Methods Engrg., 39, 1419-1431.

Chernov, A., von Petersdorff, T. \& Schwab, C. (2011) Exponential convergence of hp quadrature for integral operators with Gevrey kernels. ESAIM Math. Model. Numer. Anal., 45, 387-422.

Davies, P. \& Duncan, D. (1997) Averaging techniques for time-marching schemes for retarded potential integral equations. Appl. Numer. Math., 23, 291-310.

Davies, P. \& Duncan, D. (2003) Numerical stability of collocation schemes for time domain boundary integral equations. Computational Electromagnetics (P. J. Davies, D. B. Duncan, C. Carstensen, S. Funken, W. Hackbursh, R. Hoppe \& P. Monk eds). Berlin: Springer, pp. 51-86.

Ding, Y., Forestier, A. \& Duong, T. H. (1989) A Galerkin scheme for the time domain integral equation of acoustic scattering from a hard surface. J. Acoust. Soc. Am., 86, 1566-1572.

Dodson, S., WALKer, S. \& BluCK, M. (1997) Implicitness and stability of time domain integral equation scattering analysis. Appl. Comput. Electromagn. Soc. J., 13, 291-301. 
Erdélyi, A., Magnus, W., Oberhettinger, F. \& Tricomi, F. G. (1954) Tables of Integral Transforms. New York, Toronto, London: McGraw-Hill.

Gradshteyn, I. \& Ryzhik, I. (1965) Table of Integrals, Series, and Products. New York, London: Academic Press.

HA-Duong, T. (2003) On retarded potential boundary integral equations and their discretisation. Topics in Computational Wave Propagation: Direct and Inverse Problems. Lecture Notes in Computational Science and Engineering, vol. 31. Berlin: Springer, pp. 301-336.

Ha-Duong, T., Ludwig, B. \& Terrasse, I. (2003) A Galerkin BEM for transient acoustic scattering by an absorbing obstacle. Internat. J. Numer. Methods Engrg., 57, 1845-1882.

Hackbusch, W., Kress, W. \& SAUteR, S. (2007) Sparse convolution quadrature for time domain boundary integral formulations of the wave equation by cutoff and panel-clustering. Boundary Element Analysis (M. Schanz \& O. Steinbach eds). Berlin: Springer, pp. 113-134.

Hackbusch, W., Kress, W. \& SAUteR, S. (2009) Sparse convolution quadrature for time domain boundary integral formulations of the wave equation. IMA J. Numer. Anal., 29, 158-179.

Ismail, M. E. H. (2009) Classical and Quantum Orthogonal Polynomials in One Variable, vol. 98. Cambridge: Cambridge University Press.

KhoromskiJ, B., Sauter, S. \& Veit, A. (2011) Fast quadrature techniques for retarded potentials based on TT/QTT tensor approximation. Comput. Methods Appl. Math., 11, 342-362.

Kress, R. (1985) Minimizing the condition number of boundary integral operators in acoustic and electromagnetic scattering. Quart. J. Mech. Appl. Math., 38, 323-341.

LóPEZ-FERnÁNDEZ, M. \& SAUTER, S. (2013) Generalized convolution quadrature with variable time stepping. IMA J. Numer. Anal., 33, 1156-1175.

Lubich, C. (1994) On the multistep time discretization of linear initial-boundary value problems and their boundary integral equations. Numer. Math., 67, 365-389.

NÉDÉLEC, J. (2001) Acoustic and Electromagnetic Equations. Berlin: Springer.

RynNe, B. \& SMith, P. (1990) Stability of time marching algorithms for the electric field integral equation. J. Electromagn. Waves Appl., 4, 1181-1205.

Sauter, S. \& Schwab, C. (2011) Boundary Element Methods. Springer series in computational mathematics, Berlin Heidelberg: Springer.

SAUter, S.\& Veit, A. (2013) A Galerkin method for retarded boundary integral equations with smooth and compactly supported temporal basis functions. Numer. Math., 123, 145-176.

Veit, A. (2012) Numerical methods for time-domain boundary integral equations. Ph.D. Thesis, Universität Zürich.

Wang, X., Wildman, R., Weile, D. \& Monk, P. (2008) A finite difference delay modeling approach to the discretization of the time domain integral equations of electromagnetics. IEEE Trans. Antennas and Propagation, 56, 2442-2452.

Weile, D., Ergin, A., Shanker, B. \& Michielssen, E. (2000) An accurate discretization scheme for the numerical solution of time domain integral equations. IEEE Antennas and Propagation Society International Symposium, Salt Lake City, UT, USA, vol. 2, pp. 741-744.

Weile, D., Shanker, B. \& Michielssen, E. (2001) An accurate scheme for the numerical solution of the time domain electric field integral equation. IEEE Antennas and Propagation Society International Symposium, Boston, MA, vol. 4, pp. 516-519.

Weile, D. S., Pisharody, G., Chen, N. W., Shanker, B. \& Michielssen, E. (2004) A novel scheme for the solution of the time-domain integral equations of electromagnetics. IEEE Trans. Antennas and Propagation, 52, 283-295.

Wildman, A., Pisharody, G., Weile, D. S., Balasubramaniam, S. \& Michielssen, E. (2004) An accurate scheme for the solution of the time-domain integral equations of electromagnetics using higher order vector bases and bandlimited extrapolation. IEEE Trans. Antennas and Propagation, 52, 2973-2984. 